\title{
Synchronization of Time-Varying Networks Under Fast Switching
}

\author{
Daniel J. Stilwell, Erik M. Bollt, D. Gray Roberson
}

\begin{abstract}
To understand the role of time-varying network topologies for stability of coupled systems, we examine sometimes-coupled oscillators where the network topology that describes oscillator coupling is time-varying. We show that if the network of oscillators synchronizes for the static time-average of the topology, then the network will synchronize with the time-varying topology if the time-average is achieved sufficiently fast. Although this sufficient condition appears to be very conservative, it provides new insights about the requirements for synchronization when the network topology is time-varying. In particular, it can be shown that networks of oscillators can synchronize even if at every point in time the frozentime network topology is insufficiently connected to achieve synchronization.
\end{abstract}

\section{INTRODUCTION}

Since Huygen's early observations of weakly coupled clock pendula [24], synchronization has been found in a wide variety of phenomena, ranging from biological systems that include fire flies in the forest [11], [31], animal gates [13], descriptions of the heart [23], [46], [21], and improved understanding of brain seizures [33], to chemistry [28], nonlinear optics [47], and meteorology [16]. See one of the many excellent reviews now available, including [8], [38], [12], [44], [20], [32].

Despite the very large literature to be found, the great majority of research activities have been focused on static networks whose connectivity and coupling strengths are constant in time. For example, static networks are assumed for the analysis of [35], [36], [3]. However, there are applications where the coupling strengths and even the network topology can evolve in time. Recent work such as [43], [25], [49] are amongst the few to consider time-dependent couplings. See also [26] in which a so-called "function dynamics" gives rise to networks that evolve according to a dynamical system, somewhat similarly to our networks.

It can be argued that this work has strong connections to ad hoc communication systems and control systems on time varying networks. Fundamental connections between chaotic oscillations and proof of synchronization through symbolic dynamics [42], [37] and control [14], [9], [22] and even definition of chaos through symbolic dynamics suggest this work is rooted in a description of information flow in the network.

D. Stilwell and D. Roberson are with the Bradley Department of Electrical and Computer Engineering, Virginia Polytechnic Institute and State University, Blacksburg, VA, stilwellevt.edu, roberson@vt.edu

E. Bollt is with the Department of Mathematics and Computer Science, Clarkson Unviversity, Potsdam, NY, bollteclarckson.edu

EMB has been supported by the National Science Foundation under DMS0404778. DJS has been support by the National Science Foundation under IIS0238092 and OCE0354810 and the Office of Naval Research under N000140310444, N000140510780 and N000140510516.
Coordinated control for platoons of autonomous vehicles can also be addressed using network concepts [15], [39], [17]. Each vehicle is represented by a node, and communication or mutual sensing is represented by connections between nodes. In [17] the average position of a platoon of vehicles is regulated, and the graph Laplacian is used to describe communication between vehicles. It is shown that the spectrum of the graph Laplacian can be used to indicate stability of the controlled system. As pointed out in [39], the use of a graph Laplacian is not entirely common since it appears naturally for only a limited class of control objectives. The simplified model form explored in this paper, Eq. (3), is inspired by these problems where there seems to be a notion of average information propagation in a network.

The main result of this work comes from the fields of switched systems, and specifically builds on the concept of fast switching. Switched systems are a class of systems whose coefficients undergo abrupt change, for example, consider the linear state equation

$$
\dot{x}(t)=A_{\rho(t)} x(t)
$$

where $\rho(t): \mathbb{R} \mapsto \mathbb{Z}_{+}$is a switching sequence that selects elements from a family of matrix-valued coefficients $\Theta=$ $\left\{A_{1}, A_{2}, \ldots\right\}$. When each element of $\Theta$ is Hurwitz, stability of (1) is guaranteed if $\rho(t)$ switches sufficiently slowly. Further restrictions on elements of $\Theta$, such as existence of a common Lyapunov function, can guarantee stability for arbitrary switching functions, including those that are not slow. An excellent overview of the field of switched systems and control is presented in [30] and in the book [29].

Even when the elements of $\Theta$ are not all Hurwitz, stability of (1) is still possible, although the class of switching functions is further restricted. For example, in [48] a stabilizing switching sequence is determined by selecting elements of $\Theta$ based on the location of the state $x(t)$ in the state space. This is essentially a form of state feedback.

When no elements of $\Theta$ are Hurwitz, which is the case that is considered herein, stability of (1) can sometimes be guaranteed if the switching sequence is sufficiently fast. Loosely speaking, it can be shown that

$$
\dot{x}(t)=A_{\rho(t / \varepsilon)} x(t)
$$

is asymptotically stable if there exists a constant $T$ such that the time-average

$$
\frac{1}{T} \int_{t}^{t+T} A_{\rho(\tau)} d \tau
$$

is Hurwitz for all $t$, and if $\varepsilon$ is sufficiently small. This fact has been established in [27], [4], [45] for several classes 
of linear systems related to (2). Similar results have been presented in [2], [1] for classes of nonautonomous nonlinear systems where time is parameterized by $t / \varepsilon$ as in (2). In this case, stability of a specific average system implies stability of the original system if $\varepsilon$ is sufficiently small. In addition, this work requires the existence of a Lyapunov function that is related to a certain average of the system but which is not a function of time. This requirement is too restrictive for the class of linear time-varying systems considered herein. A new fast switching stability condition, presented in Section III, is derived in order to assess local stability of networked oscillators about the synchronization manifold.

Recent complementary results have been authored by V. Blykh, I. Belykh, and M. Hasler, in [5] and [6]. They developed a method called "connection graph stability method" whereby even for networks of time-varying connections, a bound is established based on explicitly considering the total length of all paths through edges on the network connection graph. Their bound links average path length in a way which allows them to consider a small-world regular $2 \mathrm{k}$-nearest lattice with long range connections which are switched on and off with a certain probability $p$ during short time intervals $\tau$. They also conclude synchronization thresholds not dissimilar to ours relating the switching time of the necessary (long-range) connections which must be small relative the synchronization time. However the specifics of their methods, rooted explicitly in graph theory, are different from ours, which are rooted in the field of switched systems from control engineering. Consequently, the specific details of both hypothesis and conclusions are not the same between our work and [5], [6].

\section{Preliminaries}

We consider a network of coupled oscillators consisting of $r$ identical oscillators,

$$
\dot{x}_{i}(t)=f\left(x_{i}(t)\right)+\sigma B \sum_{j=1}^{r} l_{i j}(t) x_{j}(t), \quad i=1, \ldots, r
$$

where $x_{i}(t) \in \mathbb{R}^{n}$ is the state of oscillator $i, B \in \mathbb{R}^{n \times n}$, and the scalar $\sigma$ is a control variable that sets the coupling strength between oscillators. This model is inspired by the applied questions discussed in the introduction in that it has time varying connections which still allow for enough connectionism to for global synchronization, and it is of a sufficiently simplified form to admit a complete and rigorous analysis. The scalars $l_{i j}(t)$ are elements of the graph Laplacian of the network graph and describe the interconnections between individual oscillators. Let $G(t)$ be the time-varying graph consisting of $r$ vertices $v_{i}$ together with a set of ordered pairs of vertices $\left\{v_{i}, v_{j}\right\}$ that define the edges of the graph. In this work, we assume that $\left\{v_{i}, v_{i}\right\} \in G(t)$ for $i=1, \ldots, r$. Let $\tilde{G}(t)$ be the $r \times r$ adjacency matrix corresponding to $G(t)$, then $\tilde{G}_{i, j}(t)=1$ if $\left\{v_{i}, v_{j}\right\}$ is an edge of the graph at time $t$ and $\tilde{G}_{i, j}(t)=0$ otherwise. The graph Laplacian is defined as

$$
L(t)=\operatorname{diag}(d(t))-\tilde{G}(t)
$$

where the $i^{\text {th }}$ element of $d(t) \in \mathbb{R}^{r}$ is the number of vertices that vertex $i$ is connected to, including itself. Note that solutions of (3) must be interpreted in the weak sense of Carathéodory. Indeed, the presence of a switching network leads to nonsmooth solutions, i.e. piecewise differentiable solutions which are smooth only between switching instants. For existence and uniqueness theorems for such nonlinear systems, one may refer to [18], [41].

Synchronization can be assessed by examining local asymptotic stability of the oscillators along the synchronization manifold. Linearizing each oscillator (3) about the trajectory $x^{o}(t)$, which is assumed to be on the synchronization manifold, yields

$$
\dot{z}_{i}(t)=F(t) z_{i}(t)+\sigma B \sum_{j=1}^{r} l_{i j}(t) z_{j}(t)
$$

where,

$$
z_{i}(t)=x_{i}(t)-x^{o}(t)
$$

and $F(t)=D f$ evaluated at $x^{o}(t)$. Let $L(t)$ be the $r \times r$ matrix composed of entries $l_{i j}(t)$, then the system of linearized coupled oscillators is written

$$
\begin{aligned}
\dot{z}(t) & =\left(I_{r} \otimes F(t)+\sigma\left(I_{n} \otimes B\right)\left(L \otimes I_{r}\right)\right) z(t) \\
& =\left(I_{r} \otimes F(t)+\sigma L \otimes B\right) z(t)
\end{aligned}
$$

where ' $\otimes$ ' is the Kronecker product and $z(t)=$ $\left[z_{1}^{T}(t), \ldots, z_{r}^{T}(t)\right]^{T}$. Standard properties of the Kronecker product are utilized here and in the sequel, including: for conformable matrices $A, B, C$, and $D,(A \otimes B)(C \otimes D)=$ $A C \otimes B D$. Notation throughout is standard, and we assume that $\|\cdot\|$ refers to an induced norm.

It has been shown in [35], [36] that the linearized set of oscillators (7) can be decomposed into two components: one that evolves along the synchronization manifold and another that evolves transverse to the synchronization manifold. If the latter component is asymptotically stable, then the set of oscillators will synchronize.

The claimed decomposition is achieved using a Schur transformation. We briefly describe the decomposition since it plays a central role in our assessment of synchronization under time-varying network connections. Let $P \in \mathbb{R}^{n \times n}$ be a unitary matrix such that $U=P^{-1} L P$ where $U$ is upper triangular. The eigenvalues $\lambda_{1}, \ldots, \lambda_{r}$ of $L$ appear on the main diagonal of $U$. The transformation is not unique, in that the triangular structure of $U$ can be obtained with the eigenvalues of $L$ in any order along the diagonal. A change of variables $\xi(t)=\left(P \otimes I_{n}\right)^{-1} z(t)$ yields

$$
\begin{aligned}
\dot{\xi}(t) & =\left(P \otimes I_{n}\right)^{-1}\left(I_{r} \otimes F(t)+\sigma L \otimes B\right)\left(P \otimes I_{n}\right) \xi(t) \\
& =\left(I_{r} \otimes F(t)+\sigma U \otimes B\right) \xi(t)
\end{aligned}
$$

Due to the block-diagonal structure of $I_{r} \otimes F(t)$ and the upper triangular structure of $U$, stability of (8) is equivalent to stability of the subsystems,

$$
\dot{\xi}_{i}(t)=\left(F(t)+\sigma \lambda_{i} B\right) \xi_{i}(t), \quad i=1, \ldots, r
$$


where $\lambda_{1}, \ldots, \lambda_{r}$ are the eigenvalues of $L$. Note that since the row sums of $L$ are zero, the spectrum of $L$ contains at least one zero eigenvalue. We assign $\lambda_{1}=0$, which is consistent with particular choices of the transformation matrix $P$. Thus

$$
\dot{\xi}_{1}(t)=F(t) \xi_{1}(t)
$$

evolves along the synchronization manifold, while (9) with $i=2, \ldots, r$ evolves transverse to the synchronization manifold [35]. Since the oscillators are assumed identical, the (identity) synchronization manifold is invariant for all couplings, the question being its stability. The set of coupled oscillators will synchronize if the synchronization manifold is stable, if (9) with $i=2, \ldots, r$ is asymptotically stable.

\section{MAIN RESUlT}

For a given static network, the master stability function characterizes values of $\sigma$ for which a set of coupled oscillators (7) synchronizes [35], [3], [19]. The graph Laplacian matrix $L$ has $r$ eigenvalues, which we label,

$$
0=\lambda_{1} \leq \ldots \leq \lambda_{r}=\lambda_{\max }
$$

The stability question reduces by linear perturbation analysis to a constraint upon the eigenvalues of the Laplacian:

$$
\sigma \lambda_{i} \in\left(\alpha_{1}, \alpha_{2}\right) \quad \forall i=2, \ldots, r
$$

where $\alpha_{1}, \alpha_{2}$ are given by the master stability function (MSF), a property of the oscillator equations. For $\sigma$ small, synchronization is unstable if $\sigma \lambda_{2}<\alpha_{1}$; as $\sigma$ is increased, instability arises when,

$$
\sigma \lambda_{\max }>\alpha_{2}
$$

By algebraic manipulation of (11), if,

$$
\frac{\lambda_{\max }}{\lambda_{2}}<\frac{\alpha_{2}}{\alpha_{1}}=: \beta,
$$

then there is a coupling parameter, $\sigma_{s}$, that will stabilize the synchronized state. For some networks, no value of $\sigma$ satisfies (11). In particular, since the multiplicity of the zero eigenvalue defines the number of completely reducible subcomponents, if $\lambda_{2}=0$, the network is not connected, and synchronization is not stable. However, even when $\lambda_{2}>0$, if the spread of eigenvalues is too great, then synchronization may still not be achievable.

For the case of a time-varying network topology, represented by $L(t)$, our principal contribution is to show that the network can synchronize even if the static network for any frozen value of $t$ is insufficient to support synchronization. Specifically, we show that the time-average of $L(t)$, not the frozen values of $L(t)$, is an indicator of synchronization. If the time-average of $L(t)$ is sufficient to support synchronization, then the time-varying network will synchronize if the time-average is achieved sufficiently fast.

Theorem 3.1: Suppose a set of coupled oscillators with linearized dynamics

$$
\dot{z}_{s}(t)=\left(I_{r} \otimes F(t)+\sigma \bar{L} \otimes B\right) z_{s}(t)
$$

has an asymptotically stable synchronization manifold, regarding $z(t) \rightarrow 0$ in Eq. (6). Then there exists a positive scalar $\varepsilon^{*}$ such that the set of oscillators with linearized dynamics

$$
\dot{z}_{a}(t)=\left(I_{r} \otimes F(t)+\sigma L(t / \varepsilon) \otimes B\right) z_{a}(t)
$$

and time-varying network connections $L(t)$ is also asymptotically stably synchronized, again regarding $z(t) \rightarrow 0$ in Eq. (6), for all fixed $0<\varepsilon<\varepsilon^{*}$, if there exists a constant $T$ such that $L(t)$ satisfies

$$
\frac{1}{T} \int_{t}^{t+T} L(\tau) d \tau=\bar{L}
$$

and the column sums of $L(t)$ are all zero for all $t$.

Remark 3.2: Since $L(t)$ represents a time-varying network, we may assume that for each value of $t, L(t)$ is a graph Laplacian as defined in (4). Thus the time-average $\bar{L}$ in (16) is not a graph Laplacian. In other words, $\bar{L}$ does not necessarily correspond to a particular network topology and arises only as the time-average of $L(t)$. However, $\bar{L}$ does inherit the zero row and column sum property of $L(t)$. A preliminary lemma is required to prove Theorem 3.1, the proof of which appears in the Appendix.

Lemma 3.3: Suppose there exists a constant $T$ for which the matrix-valued function $E(t)$ is such that

$$
\frac{1}{T} \int_{t}^{t+T} E(\tau) d \tau=\bar{E}
$$

for all $t$ and

$$
\dot{x}(t)=(A(t)+\bar{E}) x(t), \quad x\left(t_{o}\right)=x_{o}, \quad t \geq t_{o}
$$

is uniformly exponentially stable. Then there exists $\varepsilon^{*}>0$ such that for all fixed $\varepsilon \in\left(0, \varepsilon^{*}\right)$,

$$
\dot{z}(t)=(A(t)+E(t / \varepsilon)) z(t), \quad z\left(t_{o}\right)=z_{o}, \quad t \geq t_{o}
$$

is uniformly exponentially stable.

Proof of Theorem 3.1:

First we show that the Schur transformation that decomposes the set of oscillators (14) with static $\bar{L}$ also induces a similar decomposition for (15) with time-varying $L(t)$. Then we apply Lemma 3.3 to show that the modes of the system that evolve transverse to the synchronization manifold are stable if $\varepsilon$ is sufficiently small.

Let $P \in \mathbb{R}^{r \times r}$ be a unitary matrix such that $\bar{U}=P^{-1} \bar{L} P$ where

$$
\bar{U}=\left[\begin{array}{cc}
0 & \bar{U}_{1} \\
0_{(r-1) \times 1} & \bar{U}_{2}
\end{array}\right]
$$

is the Schur transformation of $\bar{L}$, and $\bar{U}_{2} \in \mathbb{R}^{(r-1) \times(r-1)}$ is upper triangular. Without loss of generality, we have assumed that the left-most column of $P$ is the unity norm eigenvector $[\sqrt{1 / r}, \ldots, \sqrt{1 / r}]^{T}$ corresponding to a zero eigenvalue. The change of variables $\xi_{s}(t)=(P \otimes I)^{-1} z_{s}(t)$ yields the decomposition $\xi_{s}=\left[\xi_{s 1}, \xi_{s 2}\right]^{T}$ where $\xi_{s 1} \in \mathbb{R}^{n}$, $\xi_{s 2} \in \mathbb{R}^{n(r-1)}$, and $\xi_{s 2}$ satisfies

$$
\dot{\xi}_{s 2}(t)=\left(I_{r-1} \otimes F(t)+\sigma \bar{U}_{2} \otimes B\right) \xi_{s 2}(t)
$$


As discussed in Section II, (20) is asymptotically stable by hypothesis.

We now consider the same change of variables applied to (15). First, note that

$$
U(t)=P^{-1} L(t) P=\left[\begin{array}{cc}
0 & U_{1}(t) \\
0_{(r-1) \times 1} & U_{2}(t)
\end{array}\right]
$$

since the column sums for $L(t)$ are zero for all $t$. The change of variables $\xi_{a}(t)=(P \otimes I)^{-1} z_{a}(t)$ yields the decomposition $\xi_{a}=\left[\xi_{a 1}, \xi_{a 2}\right]^{T}$ where $\xi_{a 1} \in \mathbb{R}^{n}$ evolves along the synchronization manifold and $\xi_{a 2} \in \mathbb{R}^{n(r-1)}$ evolves transverse to the synchronization manifold. To verify that the oscillators synchronize, it is sufficient to show that

$$
\dot{\xi_{a 2}}(t)=\left(I_{r-1} \otimes F(t)+\sigma U_{2}(t / \varepsilon) \otimes B\right) \xi_{a 2}(t)
$$

is asymptotically stable when $\varepsilon$ is sufficiently small. Since

$$
\bar{U}=P^{-1} \bar{L} P=\frac{1}{T} \int_{t}^{t+T} U(\tau) d \tau
$$

we conclude that

$$
\bar{U}_{2}=\frac{1}{T} \int_{t}^{t+T} U_{2}(\tau) d \tau
$$

Thus the desired result is obtained by direct application of Lemma 3.3 along with (20), (21), and (22).

\section{ILLUSTRATION}

To illustrate fast switching concepts applied to synchronization of a set of oscillators, we consider a set of $r$ Rössler attractors

$$
\begin{aligned}
& \dot{x}_{i}(t)=-y_{i}(t)-z_{i}(t)-\sigma \sum_{j=1}^{r} l_{i j}(t / \varepsilon) x_{j}(t) \\
& \dot{y}_{i}(t)=x_{i}(t)+a y_{i}(t) \\
& \dot{z}_{i}(t)=b+z_{i}(t)\left(x_{i}(t)-c\right)
\end{aligned}
$$

where $i=1, \ldots, r, a=0.165, b=0.2, c=10$, and $\sigma=0.3$. Oscillators are coupled through the $x_{i}$ variables via $l_{i j}(t)$. Coupling between subsystems (nodes) is defined by a timevarying graph $G(t)$, with corresponding adjacency matrix $\tilde{G}(t)$. The graph Laplacian $L(t)$, with entries $l_{i j}(t)$ is defined as in (4).

For the purposes of illustration, we choose a set of five graphs and corresponding adjacency matrices $\tilde{G}_{1}, \ldots, \tilde{G}_{5}$ with the property that none of them are fully connected. That is, each graph contains pairs of nodes that do not have a path between them. However, the union of vertices over all five graphs yields a fully connected graph with the longest path between nodes containing no more than two other nodes. All five graphs and the union of graph vertices are shown in Figure 1.

A simple strategy is chosen for switching among graph Laplacians associated with the set of graphs. We choose the $T$-periodic $L(t)$ defined over one period by

$$
L(t)=\sum_{i=1}^{5} L_{i} \chi_{[(i-1) T / 5, i T / 5)}(t)
$$

where $\chi_{\left[t_{1}, t_{2}\right)}(t)$ is the indicator function with support $\left[t_{1}, t_{2}\right)$. The time-average of $L(t)$ is

$$
\bar{L}=\frac{1}{\varepsilon T} \int_{0}^{\varepsilon T} L(t / \varepsilon) d t=\frac{1}{5} \sum_{i=1}^{5} L_{i}
$$

Toward computing the upper bound for $\varepsilon$ given by (36), the set of coupled oscillators (23) with coupling defined by (24) are integrated. The $x$-coordinate for each oscillator is shown in Figure 2. The $x$-coordinates clearly synchronize. Asymptotic stability of the oscillators with respect to the synchronization manifold is suggested by plotting the sumsquare deviation of the states

$$
\sum_{i=1}^{r}\left(x_{i}(t)-\mu_{x}(t)\right)^{2}+\left(y_{i}(t)-\mu_{y}(t)\right)^{2}+\left(z_{i}(t)-\mu_{z}(t)\right)^{2}
$$

about the averages

$$
\mu_{x}(t)=\frac{1}{r} \sum_{i=1}^{r} x_{i}(t)
$$

where $\mu_{y}(t)$ and $\mu_{z}(t)$ are defined similarly. Approximately exponential decay of (25) is evident in Figure 3, indicating that the oscillators synchronize.

The linear time-varying system (7) corresponding to the set of coupled Rössler attractors is computed from the Jacobian of the right-hand side of (23) evaluated at the solutions shown in Figure 2.

As described in the proof of Lemma 3.3, a Schur transformation $U$ that diagonalizes $\bar{L}$ is computed and used as a state transformation to decompose the linear time-varying system (7) into a component that evolves along the synchronization manifold and another component that evolves transverse to the synchronization manifold. The upper bound for $\varepsilon$ given in Theorem 3.1 is computed from the component of the linear system that evolves transverse to the synchronization manifold,

$$
\dot{\xi}_{a 2}(t)=\left(I_{r-1} \otimes F(t)+\sigma U_{2} \otimes B\right) \xi_{a 2}(t)
$$

We now estimate the constants $\alpha, \rho, \eta$, and $\mu$ needed to compute the right-hand side of (36) in the proof of Lemma 3.3 (see Appendix). This is used to compute an maximum value of $\varepsilon$. The constant $\alpha$ is computed from (29), while the transition matrix is computed from

$\dot{\Phi}(t, \tau)=\left(I_{r-1} \otimes F(t)+\sigma U_{2} \otimes B\right) \Phi(t, \tau), \quad \Phi(\tau, \tau)=I$

The norm of the transition matrix $\|\Phi(t, \tau)\|$ is shown in Figure 4. The initial time $\tau$ is chosen to be 40 seconds to ensure that the states of (23) are reasonably close to the synchronization manifold. An upper bound that satisfies $\|\Phi(t, \tau)\| \leq \gamma e^{-\lambda(t-\tau)}$ is also shown in Figure 4. The coefficients $\rho, \mu$ and $\eta$ in (33) are computed from $\gamma$ and $\lambda$ when evaluating the right-hand side of (36). Choosing $T=1$, the right-hand side of (36) is evaluated for this example, and we determine that the set of coupled oscillators will synchronize if $\varepsilon<3.3 \times 10^{-7}$. This shows that our bound is exceedingly conservative. For example, empirically the oscillators will synchronize with $\varepsilon=1$, as shown in Figure 5. 


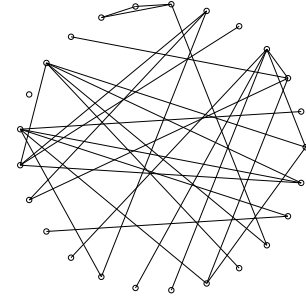

(a)

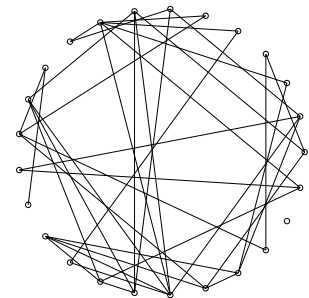

(c)

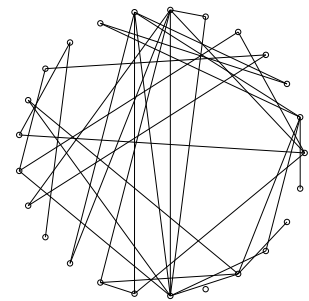

(e)

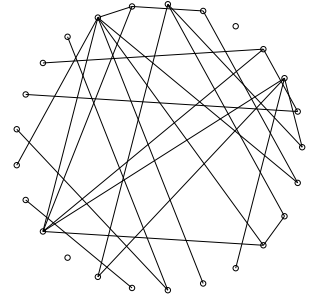

(b)

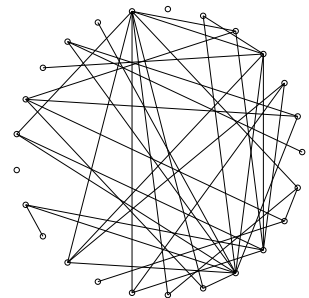

(d)

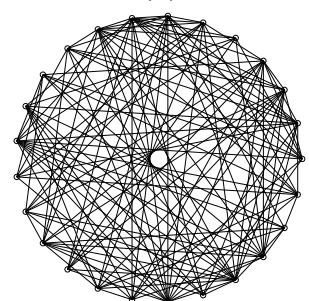

(f)
Fig. 1. (a)-(e) are graphs $G_{1}$ through $G_{5}$, respectively, while (f) is the union of graphs.

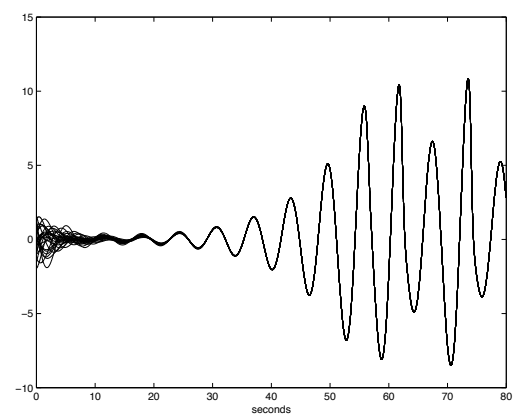

Fig. 2. The $x$-coordinate for the set of coupled Rössler attractors using the average graph Laplacian.

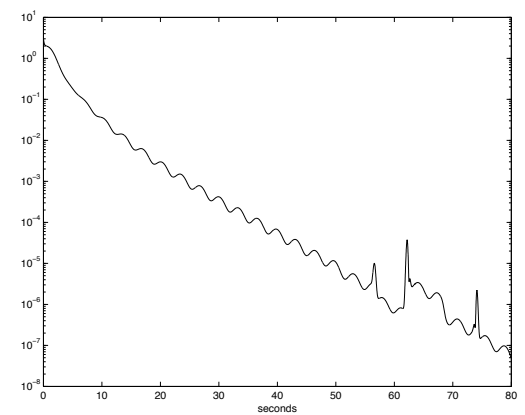

Fig. 3. Sum-square deviation in (25) for the set of coupled Rössler attractors using the average network $\bar{L}$.

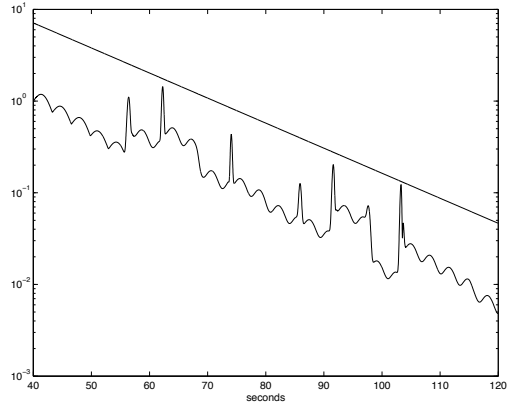

Fig. 4. Norm of the transition matrix $\Phi(t, \tau)$ along with an exponentially decaying upper bound.

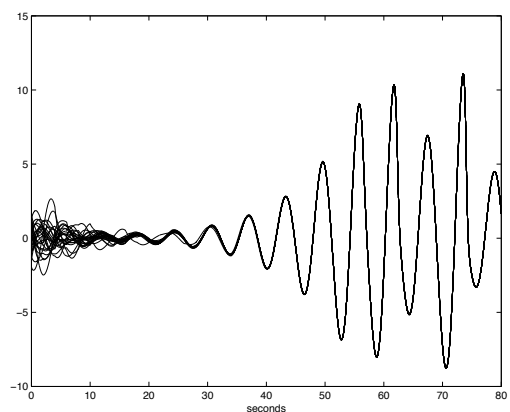

Fig. 5. The $x$-coordinate for the set of coupled Rössler attractors using the switched network where $\varepsilon=1$.

\section{APPENDIX}

Proof of Lemma 3.3

Since (18) is uniformly exponentially stable, there exists a symmetric matrix function $Q(t)$ and positive scalars $\eta, \rho$, and $\mu$ such that the Lyapunov function

$$
v(x(t), t)=x^{T}(t) Q(t) x(t)
$$

satisfies

$$
\begin{gathered}
\eta\|x(t)\|^{2} \leq v(x(t), t) \leq \rho\|x(t)\|^{2} \\
\frac{d}{d t} v(x(t), t) \leq-\mu\|x(t)\|^{2}
\end{gathered}
$$

for all $t$. To establish uniform exponential stability of (19), we will show that $v(z(t), t)$ is also a Lyapunov function for (19) if $\varepsilon$ is sufficiently small. This claim is achieved by showing that for sufficiently small values of $\varepsilon$,

$$
\Delta v(z, t+\varepsilon T, t) \equiv v(z(t+\varepsilon T), t+\varepsilon T)-v(z(t), t)
$$

is negative definite for all $t$. Expanding (28) yields

$$
\begin{aligned}
& \Delta v(z, t+\varepsilon T, t)=z^{T}(t+\varepsilon T) Q(t+\varepsilon T) z(t+\varepsilon T) \\
& \quad-z^{T}(t) Q(t) z(t) \\
& =z^{T}(t)\left(\Phi_{E}^{T}(t+\varepsilon T, t) Q(\varepsilon T+t) \Phi_{E}(t+\varepsilon T, t)-Q(t)\right) z(t)
\end{aligned}
$$

where $\Phi_{E}\left(t, t_{0}\right)$ is the transition matrix corresponding to $A(t)+E(t / \varepsilon)$, i.e., $z(t)=\Phi_{E}\left(t, t_{0}\right) z_{0}$ is the solution to (19), as discussed for example in [40]. Similarly denoting the transition matrix for $A(t)+\bar{E}$ as $\Phi_{\bar{E}}\left(t, t_{0}\right)$, we define

$$
H(t+\varepsilon T, t)=\Phi_{E}(t+\varepsilon T, t)-\Phi_{\bar{E}}(t+\varepsilon T, t)
$$


By hypothesis,

$$
\int_{t}^{t+\varepsilon T} E(\sigma / \varepsilon) d \sigma=\varepsilon T \bar{E}
$$

which implies that the Peano-Baker series representation of the transition matrices can be used to write

$$
\begin{aligned}
H(t+\varepsilon T, t)= & \sum_{i=2}^{\infty} \int_{t}^{t+\varepsilon T} A\left(\sigma_{1}\right)+E\left(\sigma_{1} / \varepsilon\right) \int_{t}^{\sigma_{1}} \ldots \\
& \ldots \int_{t}^{\sigma_{i-1}} A\left(\sigma_{i}\right)+E\left(\sigma_{i} / \varepsilon\right) d \sigma_{i} \cdots d \sigma_{1} \\
& -\sum_{i=2}^{\infty} \int_{t}^{t+\varepsilon T} A\left(\sigma_{1}\right)+\bar{E} \int_{t}^{\sigma_{1}} \cdots \\
& \ldots \int_{t}^{\sigma_{i-1}} A\left(\sigma_{i}\right)+\bar{E} d \sigma_{i} \cdots d \sigma_{1}
\end{aligned}
$$

Defining

$$
\alpha \equiv \sup _{t \geq 0}(\max (\|A(t)+\bar{E}\|,\|A(t)+E(t / \varepsilon)\|))
$$

a bound for $H(t+\varepsilon T, t)$ is computed

$$
\|H(t+\varepsilon T, t)\| \leq 2\left(e^{\varepsilon T \alpha}-1-\varepsilon T \alpha\right)
$$

Noting that $\Phi_{E}=\Phi_{\bar{E}}+H, \Delta v$ is expressed

$$
\begin{aligned}
\Delta v(z, t+\varepsilon T, t) \\
=z^{T}(t)\left(\Phi_{\bar{E}}^{T}(t+\varepsilon T, t) Q(t+\varepsilon T) \Phi_{\bar{E}}(t+\varepsilon T, t) Q(t)\right) z(t) \\
+z^{T}(t)\left(\Phi_{\bar{E}}^{T}(t+\varepsilon T, t) Q(t+\varepsilon T) H(t+\varepsilon T, t)\right. \\
\quad+H^{T}(t+\varepsilon T, t) Q(t+\varepsilon T) \Phi_{\bar{E}}(t+\varepsilon T, t) \\
\left.+H^{T}(t+\varepsilon T, t) Q(t+\varepsilon T, t) H(t+\varepsilon T, t)\right) z(t)
\end{aligned}
$$

The task now is to compute an upper bound for $\Delta v(z, t+$ $\varepsilon T, t)$ and show that this bound is negative if $\varepsilon$ is sufficiently small. Several well-known relationships that are consequences of (26), (27), and uniform exponential stability of (18) are utilized (see for example [40] pages 101 and 117, or [10], page 202). Namely,

$$
\begin{aligned}
\|Q(t)\| & \leq \rho \\
\left\|\Phi_{\bar{E}}\left(t, t_{o}\right)\right\| & \leq \sqrt{\rho / \eta} e^{-\frac{\mu}{2 \rho}\left(t-t_{o}\right)} \\
v(x(t), t) & \leq e^{-\frac{\mu}{\rho}\left(t-t_{o}\right)} v\left(x\left(t_{o}\right), t_{o}\right)
\end{aligned}
$$

for $t \geq t_{o}$

To compute an upper bound for the first term on the righthand side of (31) we note that if $x(t)=z(t)$ is chosen as the initial condition of (18) at time $t$, then

$$
\begin{aligned}
& z^{T}(t)\left(\Phi_{\bar{E}}^{T}(t+\varepsilon T, t) Q(t+\varepsilon T) \Phi_{\bar{E}}(t+\varepsilon T, t)-Q(t)\right) z(t) \\
& \quad=v(x(t+\varepsilon T), t+\varepsilon T)-v(x(t), t)
\end{aligned}
$$

From (34) and (26),

$$
\begin{aligned}
v(x(t+\varepsilon T), t+\varepsilon T)-v(x(t), t) & \leq\left(e^{-\mu \varepsilon T / \rho}-1\right) v(x(t), t) \\
& \leq \rho\left(e^{-\mu \varepsilon T / \rho}-1\right)\|x(t)\|^{2}
\end{aligned}
$$

Thus,

$$
\begin{aligned}
& z^{T}(t)\left(\Phi_{\bar{E}}^{T}(t+\varepsilon T, t) Q(t+\varepsilon T) \Phi_{\bar{E}}(t+\varepsilon T, t)-Q(t)\right) z(t) \\
& \quad \leq \rho\left(e^{-\mu \varepsilon T / \rho}-1\right)\|z(t)\|^{2}
\end{aligned}
$$

Combining (30), (32), (33), and (35) yields the desired upper bound

$$
\begin{aligned}
& \Delta v(z, t+\varepsilon T, t) \leq\left(\rho\left(e^{-\mu \varepsilon T / \rho}-1\right)\right. \\
& +4 \rho\left(\sqrt{\rho / \eta} e^{-\frac{\mu \varepsilon T}{2 \rho}}\right)\left(e^{\varepsilon T \alpha}-1-\varepsilon T \alpha\right) \\
& \left.+4 \rho\left(e^{\varepsilon T \alpha}-1-\varepsilon T \alpha\right)^{2}\right)\|z(t)\|^{2}
\end{aligned}
$$

Defining the continuously differentiable function $g(\varepsilon, x)$ to be the right-hand side of (36), it can be shown that $g(0, z)=0$ and $\frac{\partial}{\partial \varepsilon} g(0, z)=-\mu T\|z\|^{2}<0$. Thus since $g(\varepsilon, z) \rightarrow \infty$ as $\varepsilon \rightarrow \infty$, there exists $\varepsilon^{*}$ such that $g\left(\varepsilon^{*}, z\right)=0$ and $g(\varepsilon, z)<0$ for all $\varepsilon \in\left(0, \varepsilon^{*}\right)$ and $z \neq 0$. Thus $\Delta v(z, t+\varepsilon T, t)<0$ for all $\varepsilon \in\left(0, \varepsilon^{*}\right)$ and $z \neq 0$.

To show that negative-definiteness of $\Delta v(z, t+\varepsilon T, t)$ is sufficient to establish stability of (19). Choose $\varepsilon$ and $\gamma>0$ that satisfy

$$
\begin{aligned}
\Delta v\left(z, t_{o}+\varepsilon T, t_{o}\right) & =v\left(z\left(t_{o}+\varepsilon T\right), t_{o}+\varepsilon T\right)-v\left(z\left(t_{o}\right), t_{o}\right) \\
& \leq-\gamma\left\|z\left(t_{o}\right)\right\|^{2}
\end{aligned}
$$

for all $t_{o}$. From (26), $v\left(z\left(t_{o}\right), t_{o}\right) \leq \rho\left\|z\left(t_{o}\right)\right\|^{2}$, which implies that

$v\left(z\left(t_{o}+\varepsilon T\right), t_{o}+\varepsilon T\right)-v\left(z\left(t_{o}\right), t_{o}\right) \leq-(\gamma / \rho) v\left(z\left(t_{o}\right), t_{o}\right)$

Thus

$$
v\left(z\left(t_{o}+\varepsilon T\right), t_{o}+\varepsilon T\right) \leq(1-\gamma / \rho) v\left(z\left(t_{o}\right), t_{o}\right)
$$

Repeating this argument yields

$$
v\left(z\left(t_{o}+k \varepsilon T\right), t_{o}+k \varepsilon T\right) \leq(1-\gamma / \rho)^{k} v\left(z\left(t_{o}\right), t_{o}\right)
$$

for any positive integer $k$. Thus $v\left(z\left(t_{o}+k \varepsilon T\right), t_{o}+k \varepsilon T\right) \rightarrow 0$ as $k \rightarrow \infty$ which implies that $z\left(t_{o}+k \varepsilon T\right) \rightarrow 0$ as $k \rightarrow$ $\infty$. Since the limiting behavior is valid for any $t_{o}$, uniform exponential stability of (19) is established.

\section{REFERENCES}

[1] D. Aeyels and J. Peuteman, "A new aymptotic stability criteria for nonlinear time-variant differential equations," IEEE Trans. Automat. Contr., vol. 43, no. 7, pp. 968-971, 1998.

[2] D. Aeyels and J. Peuteman, "On exponential stability of nonlinear time-varying differential equations," Automatica, vol. 35, pp. 1091$1100,1999$.

[3] M. Barahona and L. M. Pecora, "Synchronization in small-world systems," Phys. Rev. Letters, 89(5), nlin.CD/0112023 (2002).

[4] R. Bellman, J. Bentsman, and S. M. Meerkov, "Stability of fast periodic systems," IEEE Trans. Automat. Contr., vol. 3, no. 30, pp. 289-291, 1985.

[5] V. N. Belykh, I. V. Belykh, M. Hasler, "Connection graph stability method for synchronized coupled chaotic systems," 195 159-187 (2004).

[6] I. V. Belykh, V. N. Belykh, M. Hasler, "Blinking model and synchronization in small-world networks with a time-varying coupling," Physica D 195 188-206 (2004).

[7] I. I. Blekman, Synchronization in Science and Technology, Nauka, Moscow, 1981 (in Russian); ASME Press, New York, 1988 (in English). 
[8] S. Boccaletti, J. Kurths, G. Osipov, D. L. Valladares, and C. S. Zhou , "The synchronization of chaotic systems," Physics Reports 366 (2002), $1-101$.

[9] E. M. Bollt, "Review of chaos communication by feedback control of symbolic dynamics," Int. J. Bifurcation and Chaos 13(2), pp. 269-285 (2003).

[10] R. W. Brockett, Finite Dimensional Linear Systems. New York, NY: John Wiley and Sons, 1970.

[11] J. Buck and E. Buck, "Synchronous fireflies," Sci. Am. 234, 74 (1976).

[12] G. Chen and Y. Xinghuo, "Chaos Control Theory and Applications Series: Lecture Notes in Control and Information Sciences," Springer Series 292 (2003).

[13] J. J. Collins and I. Stewart, "Coupled nonlinear oscillators and the symetries of animal gaits," Science 3, 3, 349-392 (1993).

[14] N. J. Corron, S. D. Pethel, and K. Myneni, "Synchronizing the information content of a chaotic map and flow via symbolic dynamics,", Phys. Rev. E, 66, 036204 (2002).

[15] J. P. Desai, J. P. Ostrowski, and V. Kumar, "Modeling and Control of Formations of Nonholonomic Mobile Robots", IEEE Trans. Robotics and Automation, vol. 17, no. 6, pp. 905 - 908, 2001.

[16] G. S. Duane, P. J. Webster, and J. B. Weiss, "Go-occurrence of Northern and Southern Hemisphere blocks as partially synchronized chaos," J Atmos Sci, 56 (24), 4183-4205 (1999).

[17] J. A. Fax and R. M. Murry,"Information Flow and Cooperative Control of Vehicle Formations", IEEE Trans. Automatic Control, vol. 49, no. 9, pp. $1465-147,2004$

[18] A. F. Filippov, Differential Equations with Discontinuous Right-Hand Sides. Dordrecht, the Netherlands: Kluwer Academic Publishers, 1988.

[19] K. Fink, G. Johnson, T. Carroll, D. Mar, L. Pecora, Physical Review E, vol. 61;5, 2000.

[20] L. Glass and M. C. Mackey, From Clocks to Chaos: The Rythms of Life, Princeton U. Press (1988).

[21] M. R. Guevara, A. Shrier, and L. Glass. "Phase-locked rhythms in periodically stimulated heart cell aggregates," American Journal of Physiology, 254 (Heart Circ. Physiol. 23), H1-H10 (1988).

[22] S. Hayes, C. Grebogi, and E. Ott, "Communicating with chaos," Phys. Rev. Lett. 70(20), pp. 3031-3034 (1993).

[23] J. Honerkamp, "The heat as a system of coupled nonlinear oscillators," J. Math. Bio., 19, 69-88 (1983).

[24] C. Hugenii, Horoloquium Oscilatorium, Apud F. Muguet, Parisiis, 1673.

[25] J. Ito amd K. Kaneko, "Spontaneous structure formation in a network of chaotic units with variable connection strengths," Phys. Rev. Lett., 88 (2002) 028701.

[26] N. Kataoka and K. Kaneko, "Dynamical networks in function dynamics," Physica D, 181, pp. 235-251 (2003).

[27] R. L. Kosut, B. D. O. Anderson, and I. M. Y. Mareels, "Stability theory for adaptive systems: Method of averaging and persistency of excitation," IEEE Trans. Automat. Contr., vol. 1, no. 32, pp. 26-34, 1987.

[28] Y. Kuramoto, Chemical Oscillations, Waves and Turbulence, Springer:Berlin (1984).

[29] D. Liberzon, Switching in Systems and Control. Boston: Birkhauser, 2003.

[30] D. Liberzon and A. S. Morse, "Basic problems in stability and design of switched systems," IEEE Control Systems, vol. 5, no. 19, pp. 59-70, 1999.

[31] R. Mirollo and S. Strogatz, "Synchronization of pulsed-coupled biological oscillators," SIAM J. Appl. Math 50, 6, 1645-1662 (1990).

[32] E. Mosekilde, Y. Maistrenko, and D. Postnov, Chaotic Synchronization: Applications to Living Systems, World Scientific Nonlinear Science Series A (2002).

[33] T. I. Netoff and S. J. Schiff, "Decreased Neuronal Synchronization During Experimental Seizures," Journal of Neuroscience, 22: 7297 7307, 2002.

[34] T. Nishikawa, A. E. Motter, Y.-C. Lai and F. C. Hoppensteadt, "Heterogeneity in oscillator networks: Are smaller worlds easier to synchronize?" cond-mat/0306625.

[35] L. M. Pecora and T. L. Carroll, "Master stability function for synchronized coupled systems," Physical Review Letters, vol. 80, no. 10, pp. 2109 - 2112, 1998.

[36] L. Pecora, T. Carroll, G. Johnson, D. Mar, and K. S. Fink, "Synchronization stability in coupled oscillator arrays: Solution for arbitrary configurations," International Journal of Bifurcation and Chaos, vol. 10, no. 2, pp. $273-290,2000$.
[37] S. D. Pethel, N. J. Corron, Q. R. Underwood, and K. Myneni, "Information flow in chaos synchronization: Fundamental tradeoffs in precision, delay, and anticipation," Phys. Rev. Lett., 90, 254101 (2003).

[38] A. Pikovsky, M. Rosemblum, and J. Kurths, Synchronization, A Universal Concept in Nonlinear Sciences, Cambridge University Press:Cambridge (2001).

[39] D. G. Roberson and D. J. Stilwell, "Control of an Autonomous Underwater Vehicle Platoon with a Switched Communication Network", Proceedings of the American Control Conference, Portland, OR, 2005.

[40] W. J. Rugh, Linear Systems Theory, Second Edition. Upper Saddle River, NJ: Prentic Hall, 1996.

[41] G. Sansone and R. Conti, Non-LinearDifferential Equations. Oxford, England: Pergamon Press Inc., 1964.

[42] T. Stojanovski, L. Kocarev, and R. Harris , "Applications of symbolic dynamics in chaos synchronization," IEEE Trans. Circuits and Systems I: Fundamental Theory and Applications, 44(10), (1997).

[43] T. Stojanovski, L. Kocarev, U. Parlitz and R. Harris, "Sporadic driving of dynamical systems," Phys. Rev. E, 55 (4), pp. $4035-4048$ (1997).

[44] S. Strogatz, Sync: The Emerging Science of Spontaneous Order, Hyperion, (2003)

[45] J. Tokarzewski, "Stability of periodically switched linear systems and the switching frequency," International Journal of Systems Science, vol. 4, no. 18, pp. 697-726, 1987.

[46] V. Torre, "A theory of synchronization of two heart pacemaker cells," J. Theor. Bio., 61, 55-71 (1976).

[47] G. D. Van Wiggeren and R. Roy, Science 279 (1998) 1198; R. Roy, K. S. Thornburg Jr., Phys. Rev. Lett. 72 (1994) 2009; J. Ohtsubop, "Feedback Induced Instability and Chaos in Semiconductor Lasers and Their Applications," Optical Review, 6 (1), (1999), 1-15.

[48] M. Wicks, P. Peleties, and R. DeCarlo, "Switched controller synthesis for the quadratic stabilization of a pair of unstable linear systems," European Journal of Control, vol. 4, pp. 140-147, 1998.

[49] D. H. Zanette and A. S. Mikhailov, "Dynamical systems with timedependent coupling: clustering and critical behavior", Physica D, 194, pp. 203-218 (2004). 